\title{
REPLY
}

\section{Renal denervation-a valid treatment option despite SYMPLICITY HTN-3}

Roland E. Schmieder

I thank Yutang Wang for his Correspondence (Ethnicity and sympathetic tone: predictors of the blood pressure response to renal denervation? Nat. Rev. Cardiol. doi:10.1038/nrcardio.2014.70-c1 $)^{1}$ on my News \& Views article (How should data from SYMPLICITY HTN-3 be interpreted? Nat. Rev. Cardiol. 11, 375-376; 2014). ${ }^{2}$ Wang highlights 11 studies with low-level evidence and a small number of patients $(n=6-17)$ in which the efficacy of renal denervation for lowering blood pressure was examined. Although Wang acknowledges these study limitations, conclusions are still drawn from these reports. In contrast to these small studies, Mahfoud et al. conducted a large, prospective study involving 206 patients, and observed significant decreases in office and ambulatory blood pressure with renal denervation after 6 months. ${ }^{3}$ Data from the SYMPLICITY HTN-1 trial, ${ }^{4}$ and from the randomized, controlled SYMPLICITY HTN-2 trial, ${ }^{5}$ both published in 2014, have also demonstrated significant decreases in office blood pressure with renal denervation after 3 years.

In my News \& Views article, ${ }^{2}$ I aimed to interpret the results from the SYMPLICITY HTN-3 trial, ${ }^{6}$ a prospective, randomized, masked, single-blind study that did not show a significant blood pressure reduction after renal denervation. In my opinion, some of the viewpoints expressed by Wang are not correct. Studies with witnessed intake of medication to exclude patients with poor drug adherence have been published; ${ }^{1}$ however, this observation would not help in the interpretation of the SYMPLICITY HTN-3 data, ${ }^{6}$ because poor drug adherence would have affected both the renal denervation and shamcontrolled groups. Adherence to medical therapy in treatment-resistant hypertension is a serious obstacle. A study involving 79 patients with hypertension demonstrated a nonadherence rate of $25 \%$ and $23 \%$ at baseline and 6 months, respectively, after renal denervation, where adherence was assessed by toxicological urine measurements. ${ }^{7}$ Drug adherence was not verified in the SYMPLICITY HTN-3 trial, ${ }^{6}$ which is a serious limitation.

Additional limitations related to procedural discrepancies that call into question the overall validity of the SYMPLICITY HTN-3 data have since been reported. ${ }^{6}$ Bhatt and Bakris wrote that "each procedure was supervised by an experienced doctor performed per protocol instruction. If those instructions did not allow for aggressive enough denervation owing to an insufficient number of ablations, lack of four-quadrant ablations, or other technical features, that might explain the failure to lower blood pressure significantly." ${ }^{8}$ This statement is most irritating. If technical performance jeopardized the quality of the renal denervation intervention in the SYMPLICITY HTN-3 trial, interpretation of the whole study becomes difficult. Indeed, four-quadrant ablation was achieved on both renal sites in only 19 patients, and in one of the two sites in 68 patients. The aim of each renal-denervation procedure is to achieve a full circumferential (360 grade) ablation by applying high-frequency energy at the superior, inferior, anterior, and posterior arterial walls. However, in the SYMPLICITY HTN-3 trial, 253 out of 340 renal-denervation procedures did not have a complete four-quadrant ablation on either site. ${ }^{9}$ The reductions in office blood pressure and ambulatory blood pressure were $-24.3 \mathrm{mmHg}$ and $-10.3 \mathrm{mmHg}$ in those receiving two four-quadrant ablations, respectively; $-16.7 \mathrm{mmHg}$ and $-7.7 \mathrm{mmHg}$ in those receiving one four-quadrant ablation, respectively; and $-14.2 \mathrm{mmHg}$ and $-6.3 \mathrm{mmHg}$ in those who did not receive a four-quadrant ablation on either site, respectively (data presented at the EuroPCR 2014 by D. Kandzari and at the European Hypertension Meeting 2014 by G. Bakris).
Therefore, we now have evidence that the performance goal of a circumferential renal ablation was not achieved in $69 \%$ of the renal-denervation procedures. This consideration clearly limits the interpretation of the trial data (including the ethnicity results $^{6}$ ) and, therefore, we cannot conclude from the SYMPLICITY HTN-3 trial results that renal denervation is not effective for lowering blood pressure. ${ }^{1}$

What next? First, we need more rigorous prospective, randomized, sham-controlled studies to evaluate the precise effectiveness of catheter-based bilateral renal denervation in patients with treatment-resistant hypertension. Multi-electrode devices could be redesigned to improve efficacy, and selection of operators that have substantial experience in performing renal denervation would decrease the number of incomplete renal-denervation procedures. Second, an urgent need exists to identify clinical predictors of the blood-pressure response to renal denervation. A reduction in systolic office blood pressure $>10 \mathrm{mmHg}$ occurred in $72-84 \%$ of patients in studies with inclusion criteria similar to those in the SYMPLICITY studies. ${ }^{4,10}$ Third, after clinical predictors have been identified, their usefulness in clinical practice will need to be proven by outcome research trials, such as the GLOBAL registry. ${ }^{11}$

Clinical Research Unit of Hypertension and Vascular Medicine, Department of Nephrology and Hypertension, University Hospital Erlangen, Krankenhausstraße 12, 91054 Erlangen, Germany.

roland.schmieder@uk-erlangen.de

\section{Competing interests}

The author has received speaker fees, consultancy fees, and advisory board fees from Boston Scientific, Kona Medical, Medtronic, Recor, and Terumo. The University Hospital has received research funding from Medtronic and Rox Medical.

1. Wang, Y. Ethnicity and sympathetic tone: predictors of the blood pressure response to renal denervation? Nat. Rev. Cardiol. http:// dx.doi.org/10.1038/nrcardio.2014.70-c1. 


\section{CORRESPONDENCE}

2. Schmieder, R. E. Hypertension: How should data from SYMPLICITY HTN-3 be interpreted? Nat. Rev. Cardiol. 11, 375-376 (2014).

3. Mahfoud, F. et al. Ambulatory blood pressure changes after renal sympathetic denervation in patients with resistant hypertension. Circulation 128, 132-140 (2013).

4. Krum, H. et al. Percutaneous renal denervation in patients with treatment-resistant hypertension: final 3-year report of the Symplicity HTN-1 study. Lancet 383, 622-629 (2014).

5. Esler, M. D. et al. Catheter-based rena denervation for treatment of patients with treatment-resistant hypertension: 36 month results from the SYMPLICITY HTN-2 randomized clinical trial. Eur. Heart J. 35, 1752-1759 (2014).

6. Bhatt, D. L. et al. A controlled trial of renal denervation for resistant hypertension. N. Engl. J. Med. 370, 1393-1401 (2014).

7. Schmieder, R. E. et al. Adherence to antihypertensive medication in treatment resistant hypertension: baseline data and 6 months follow-up after renal denervation. Presented at the Joint Meeting of the European Society of Hypertension and International Society of Hypertension 2014.
8. Bhatt, D. L. \& Bakris, G. L. Renal denervation for resistant hypertension. N. Engl. J. Med. 371 , 184 (2014).

9. Kandzari, D. E. Symplicity HTN-3: insights from the subgroup analysis. Presented at the EuroPCR 2014 Hot Line Session.

10. Persu, A. et al. Blood pressure changes after renal denervation at 10 European expert centers. J. Hum. Hypertens. 28, 150-156 (2014)

11. Pathak, A. et al. From SYMPLICITY HTN-3 to the Renal Denervation Global Registry: where do we stand and where should we go? Eurolntervention 10, 21-23 (2014). 